\title{
WIRELESS LANDMINE DETECTION ROBOT
}

\author{
VRISHALI PATIL ${ }^{1} \&$ UTTAM BOMBALE $^{2}$ \\ ${ }^{I}$ Post Graduate Scholar, Department of Technology, Shivaji University, Kolhapur, Maharashtra, India \\ ${ }^{2}$ Associate Professor, Department of Technology, Shivaji University, Kolhapur, Maharashtra, India
}

\begin{abstract}
In this paper, I have proposed a system of wireless landmine detection robot. For that various devices and modules are used such as Zigbee, sensors, controller, motors and drivers etc. Wireless locomotion of the robot is achieved using remote control unit and control unit. Wireless data are transmitted through a Zigbee module. Zigbee is configured using digi XCTU software. Depending upon the moment of joystick on a remote control module controller selects the binary code and send it to driver. Pulse induction type metal detector is used as it has high accuracy and depth of penetration. GPS sensor is located on robot side to give the location of a robot in the form of longitude and latitude.

KEYWORDS: Land Mine Detection, Wireless Robot \& Landmine Detection Sensors
\end{abstract}

Received: Mar 23, 2019; Accepted: Apr 13, 2019; Published: Apr 27, 2019; Paper Id.: IJRRDJUN20194

\section{INTRODUCTION}

National security is of prime importance in today's weapon studded world and therefore the need to consider the safety of the army personnel and people living in war prone areas becomes very vital. A Landmine is basically an explosive device hidden underground by the enemy and explodes when any personnel or vehicle steps or drives over it. The Pressure created by the personnel or the vehicle on the ground below which the mine is laid acts as the detonator for the mine explosion. The damage caused by the Landmine explosion is fatal and hence detecting landmines becomes necessary before the army personnel or vehicle accidentally steps over it. The major challenge is detecting these landmines without causing any explosion and diffusing them once they are detected. The process of detecting landmines is technically termed as minesweeping and process of removing or defusing the mines is known as demining or mine clearance. Minesweeping was earlier done using trained animals like dogs and rats but modern methods includes metal detectors and various tooled attached to the vehicles. But any manual intervention of a human is always dangerous.

Robots are used for various applications in industrial area. Robot performs various activities and is becoming more advanced. That's the reason nowadays Landmine Detection Robotic Vehicles and unmanned robots are used to detect the landmines. Robots are always reliable in terms of perfection in detection and no human life is endangered in the process.

\section{LITERATURE SURVEY}

A landmine is a type of self-contained explosive device which is placed onto or into the ground, exploding when triggered by a vehicle, a person, or an animal. The name originates from the practice of sapping, where tunnels were dug under opposing forces or fortifications and filled with explosives.

Rasaq Bello, Literature Review on Landmines and Detection Methods [2]. This paper has a review on 
landmines and detection methods, various types of landmine are explained in details such as Anti personnel, Anti tank, Common anti personnel. Various landmine detection techniques mentioned in this paper are:

Back Scattered X-Ray Radiography: In this method detection of landmines is done using backscattered x-rays with the help of collimated x-ray beam and uncollimated detectors. This system works under various weather conditions.

- Penetrating Radiation: This method is useful for material characterization of explosive materials but in case of landmine detection requires the access to two opposite sides of an object, which is difficult to obtain in case of landmines.

- Ultrasound Techniques: Properties of an ultrasonic pulse which can propagate through the material are measured through which the mechanical characteristics of the material are measured.

- Acoustic Technique: It's a non-destructive testing method using flaw detection and material characterization, principle area of which is landmine detection.

- Thermography: thermal signature of the soil is altered by the presence of shallowly buried objects is the main principle behind this method. Since landmines are shallowly buried objects this method is a topic of great interest in past few years.

- Ground Penetrating Radars: Short Pulse, wide band low energy radars are used to probe into the earth, but this method has a problem that the dielectric discontinues at the places other than the mine.

\section{Waqar Farooq, Nehal Butt, Sameed Shukat, Nouman Ali Baig, Sheikh Muhammad Ahmed, Wirelessly}

Controlled Mine Detection Robot [3]. This paper demonstrates the problem and effects of landmines in defence fields. The robot is equipped with special wheels controlled by H-Bridge module, allowing it to move in all possible directions. The robot is equipped with special range sensors that help in avoiding the obstacles in the field by specifically detecting the position of obstacles. A special type of prototype made of lightweight temperature resistant metal is used to carry all objects. A wireless camera is added to the robot, which captures and broadcasts the present location of the robot.

Jebasingh Kirubakaran. S. J, Anish Kumar Jha, Dheeraj Kumar, Sadambi Poornachandran Prakash, Mine Detecting Robot with Multi Sensors Controlled Using HC-12 Module [4]. This paper demonstrates a mine detecting robot guided by $\mathrm{HC}-12$ module that allows it to scan the testing area within $1.8 \mathrm{~km}$ and metal detector is used as mine detecting sensor placed in front of the vehicle, while GPS is used to provide the exact location of the infected area. The embedded system is based on Arduino technologies and guided by an HC -12Module.

V. Abilash and J. Paul Chandra Kumar, Arduino Controlled Landmine Detection Robot [5]. This paper demonstrates a prototype model of a land-mine detection robot which can be operated remotely using Wi-Fi. Technology. A Global Positioning System (GPS) sensor is employed, which identifies and broadcasts the present location of the robot. Path planning, obstacle detection and avoidance algorithms were used to control accurately and to navigation of the proposed path by avoiding obstacles. Arduino microcontroller is employed in this robot.

Bharath J, Automatic Land Mine Detection Robot Using Microcontroller [6]. This paper demonstrates the landmine detection robot. In this he metal detector circuit is interfaced with the robot and it is left on the required search area in order to detect the metallic components used in the landmines. 
Michael YU. Rachkov, Lino Marques, Anibal T. De Almeida, Multi-Sensing Demining Robot [7]. The paper describes an advanced multisensory demining robot. The robot transport system is based on a simple structure using pneumatic drive elements. The robot has a robust design and can carry demining equipment up to $100 \mathrm{~kg}$ over rough terrains. Due to the adaptive possibilities of pedipulators to obstacles, the robot can adjust the working position of the demining sensors while searching for mines. The detection block consists of a metal detector, an infrared detector, and a chemical explosive sensor. The robot is controlled by means of an on-board processor and by an operator remote station in an interactive mode. Experimental results of the transport, control, and detection systems of the robot are presented.

Seong Pal Kang, Junho Choi, Seung-Beum Suh, Sungchul Kang, Design of mine detection robot for Korean mine field [8]. The design includes a track type main platform with a simple moving arm and a mine detection sensor (consists of a metal detector and a GPR at this stage). In addition, in order to maintain the effective distance between the landmine sensors and ground surface, a distance sensing technique for terrain adaptability was developed and briefly introduced in this paper.

Zhenjun He, Jiang Zhang, Peng Xu, Jiaheng Qin and Yunkai Zhu, Mine Detecting Robot Based on Wireless Communication with Multi-sensor[9]. This paper put forward a control method and device of robot, Robot based on wireless communication with multi-sensor, which can carry out robot's motion control, video surveillance, temperature and humidity detection, gas detection and data exchange, etc. In a timely manner, it can also upload real-time monitoring information to the upper machine to carry on the dynamic measurement and real-time monitoring, and give security alerts.

\section{Majd Ghareeb, Ali Bazzi, Mohamad Raad, Samih Abdul Nabi Wireless. Robo-Pi for Landmine Detection} [10]. The main idea is to design and implement a prototype of an efficient low cost automated mine detector that will replace the current employed human detectors in the mission of detecting and extracting mines in a suspected area of land. This detector will wirelessly communicate with a server to transmit the detected information such as the location of the metal object and captured images of the land where does it exist.

\section{Proposed System}

The wireless landmine detection robot is the integration of three modules such as a control unit, remote and sensors.

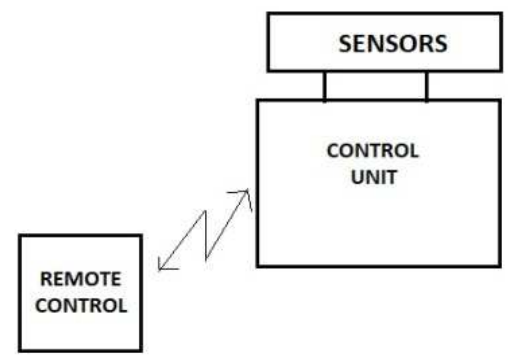

Figure 1

\section{Remote Control}

This unit consist of Arduino, joystick, LCD screen and Zigbee S2C (co-ordinator). Arduino is used to control the functions of joystick and Zigbee. Joystick decides the direction of the robot. These instructions are wirelessly transferred to control unit. If landmine found gps location will be displayed on LCD screen. 


\section{Control Unit}

It consist of Zigbee S2C (end device), motor driver L298N and 12 volt battery. The commands are received from remote control through control unit Zigbee. Depending upon the commands Arduino sets the binary logic and transmits it to motor driver. Motor Driver energies the motor accordingly and robot changes its position. Arduino receives signals from various sensors.

\section{Sensors}

Metal Detector sensor and GPS sensors are main sensors in the landmine detection robot. Here pulse induction type metal detector is used. Metal Detector senses the underground object and generate the high pulse at the output which was received by Arduino. As soon as high pulse is received Arduino commands GPS sensor to send the longitude and latitude of the location. Which is further sent to remote control to display on LCD screen. Control unit and various sensors are mounted on robot chassis only

\section{BLOCK DIAGRAM}

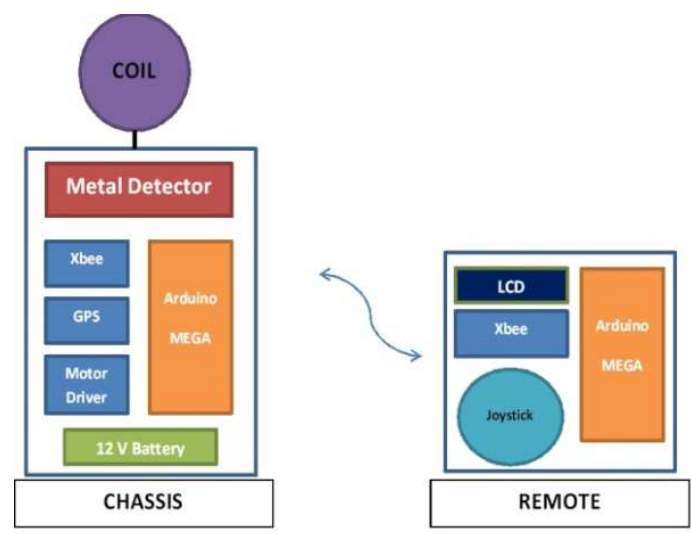

Figure 2

\section{Metal Detector}

In this project pulse induction type metal detector is used. It has a deep range of metal detection. Basically op amp as oscillator circuit is used. Pulse input is given to controller. Output is collected in the form of buzzer as well given to Arduino mega.

\section{Zigbee Module}
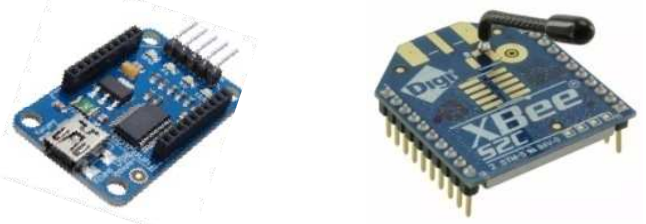

Figure 3

For wireless transmission of data from remote to robot and vice versa wireless module is used. Here ZigbeeTX Rx pair is used as wireless module. Zigbee S2C model is used. It gives a 100m range. But if want to improve the range we can use Zigbee pro which gives about range of $1 \mathrm{Km}$. Zigbee pair is converted one to co-ordinator and other to end device. 
Co-ordinator is placed at remote side and end device on robot. We can communicate with multiple robots with single co-ordinator.

\section{GPS Module}

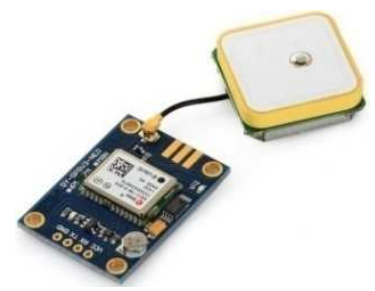

Figure 4

To give the location of Landmine, GPS module is used. It gives the location in terms of longitude and latitude form. This device can instantaneously give your exact position almost anywhere on the planet. GPS receivers have tiny processors and antennas that directly receive the data sent by the satellites and compute your position. I have used NEO-6M GPS module. It is a complete GPS module which uses the latest technology from to give the best possible positioning information and includes a larger built-in 25 x $25 \mathrm{~mm}$ active GPS antenna with a UART TTL socket. This is an updated GPS module that can be used with ardupilot mega v2.

The antenna is connected to the module through a ufl cable which allows for flexibility in mounting the GPS such that the antenna will always see the sky for best performance. This makes it powerful to use with cars and other mobile applications. The GPS module has serial TTL output, it has four pins: TX, RX, VCC, and GND.

\section{Motor Driver}

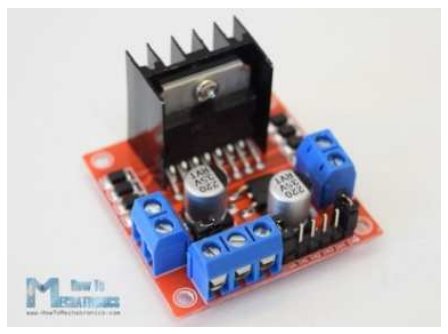

Figure 5

The L298N is a dual H-Bridge motor driver which allows speed and direction control of two DC motors at the same time. The module can drive DC motors that have voltages between 5 and $35 \mathrm{~V}$, with a peak current up to $2 \mathrm{~A}$.

\section{Controller}

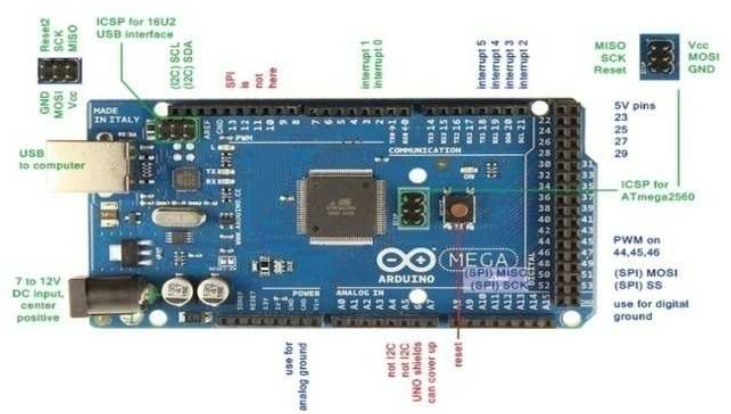

Figure 6 
The Arduino Mega is a microcontroller board based on the ATmega2560. It has 54 digital input/output pins, 16 analog inputs, 4 UARTs (hardware serial ports), a $16 \mathrm{MHz}$ crystal oscillator, a USB connection, a power jack, an ICSP header, and a reset button. It contains everything needed to support the microcontroller; simply connect it to a computer with a USB cable or power it with a AC-to-DC adapter or battery to get started. We have used this both remote and robot side.

\section{LCD}

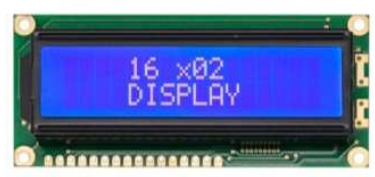

Figure 7

$16 \times 2$ LCD is named so because; it has 16 Columns and 2 Rows. There are a lot of combinations available but the most used one is the $16 \times 2$ LCD. So, it will have $(16 \times 2=32) 32$ characters in total and each character will be made of $5 \times 8$ Pixel Dots.

\section{Joystick}

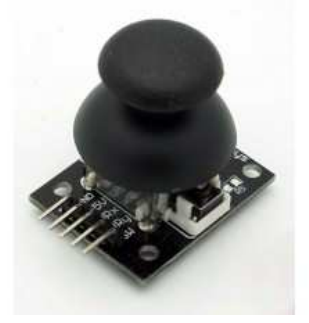

Figure 8

Joystick is placed at remote side to give forward, left, right instruction. In this joystick directional movements are simply two potentiometers - one for each axis. Pots are $\sim 10 \mathrm{k}$ each. This joystick also has a select button that is actuated when the joystick is pressed down. The Joystick module has $\mathrm{X}, \mathrm{Y}$ analog outputs and has a switch that detects button press. The joystick is made up of two passive potentio-meters (variable resistors) and a push button and therefore can be used at any standard voltage levels

\section{Sources}

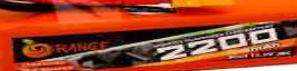

\section{Figure 9}

5 volt and 12 volt power supply batteries are used to energies the whole circuitry. We have used lithium polymer battery of 12volt $2200 \mathrm{mAH}$. It is rechargeable 


\section{RESULTS}

\section{Zigbee Configuration}

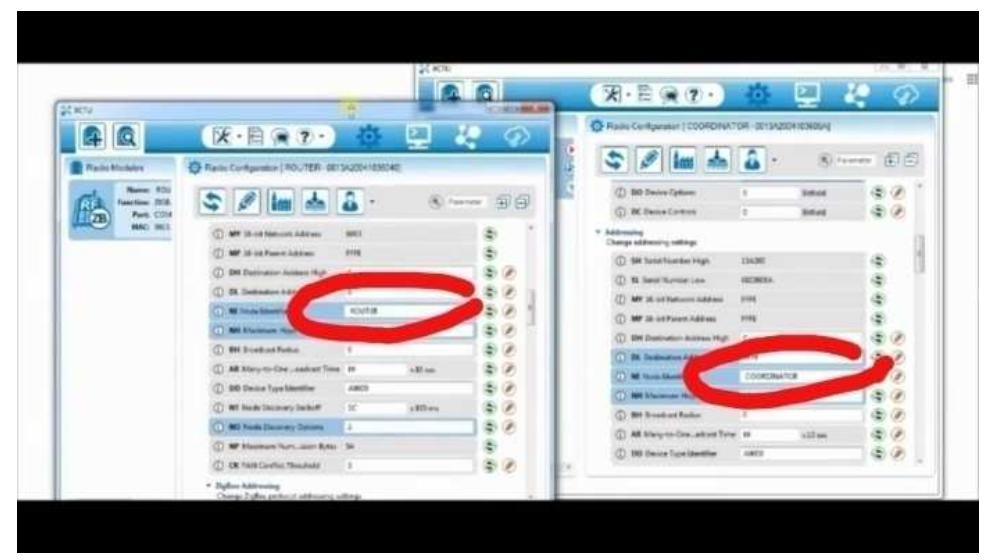

Figure 10

Zigbee S2C model is used which need to configure in AT mode. One Zigbee is configured as co-ordinator other as router. For configuration XCTU software is used.

\section{GPS Module Testing}

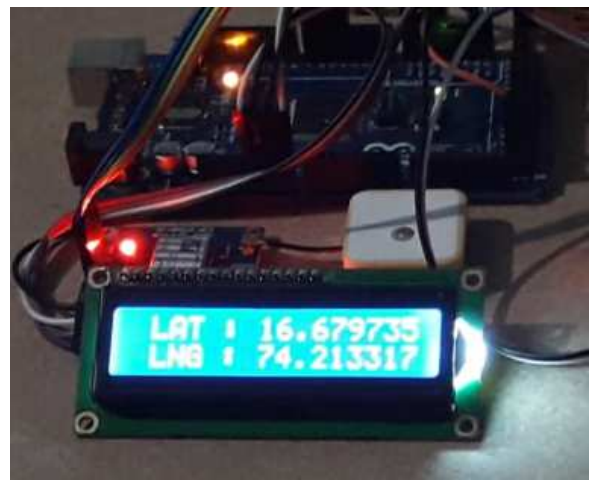

Figure 11

GPS module is programmed through Arduino mega and accurate gps location is temporally displayed on lcd for testing purpose.

\section{Robot}

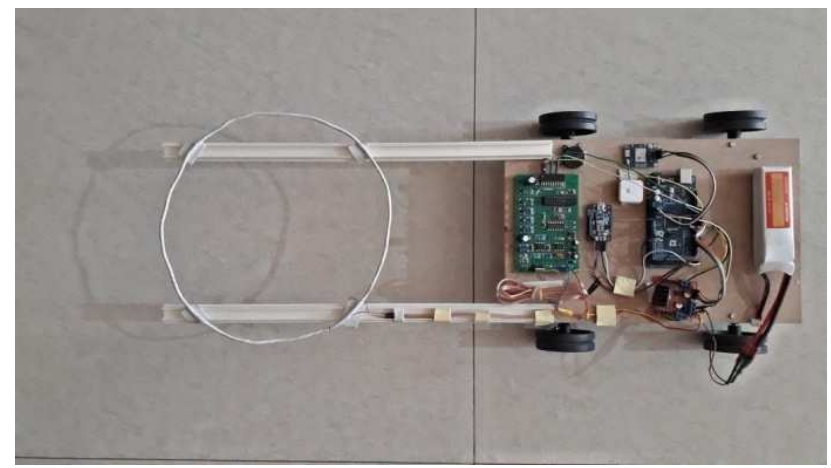

Figure 12 


\section{Remote}

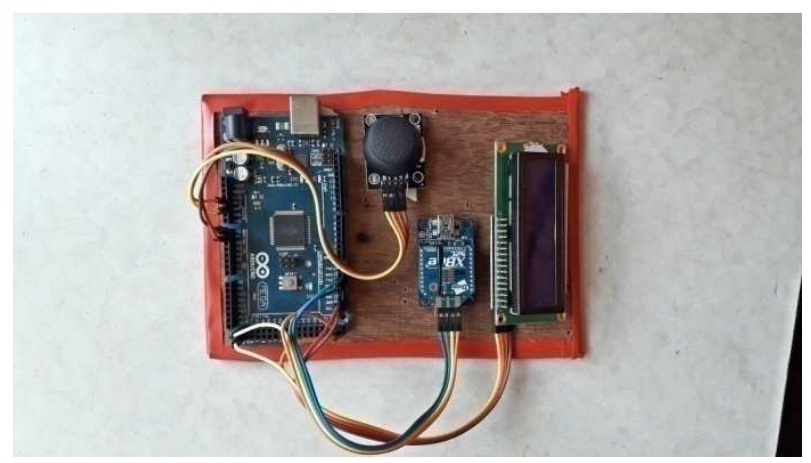

Figure 13

\section{CONCLUSIONS AND FUTURE SCOPE}

In this paper the overall design of wireless landmine detection robot has described. We have tried to make the system less expensive and compact. The future scope for this project is the robot model can be modified with drone. Instead on metal detector GPR module can implemented. Some demining features can be added. Also we can use colour spray at robot side to mark the probable landmine location.

\section{REFERENCES}

1. L. Robledo, M. Carrasco and D. Mery, " A survey of land mine detection technology” International Journal of Remote Sensing Vol. 30, No. 9, 10 May 2009, 2399-2410

2. Rasaq Bello, "Literature Review on Landmines and Detection Methods" Frontiers in Science.

3. Waqar Farooq, Nehal Butt, Sameed Shukat, Nouman Ali Baig, Sheikh Muhammad Ahmed, "Wirelessly Controlled Mine Detection Robot"2016 International Conference on Intelligent Systems Engineering (ICISE)

4. Jebasingh Kirubakaran. S. J, Anish Kumar Jha, Dheeraj Kumar, Sadambi Poornachandran Prakash, "Mine Detecting Robot with Multi Sensors Controlled Using HC-12 Module ”International Journal of Engineering \& Technology

5. V. Abilash and J. Paul Chandra Kumar, "Arduino Controlled Landmine Detection Robot" 2017 Third International Conference On Science Technology Engineering and Management (ICONSTEM)

6. Bharath J, “Automatic Land Mine Detection Robot Using Microcontroller”, International Journal of Advance Engineering and Research Development Volume 4, Issue 3, March-2017

7. Seong Pal Kang, Junho Choi, Seung-Beum Suh, Sungchul Kang, "Design of mine detection robot for Korean mine field.”

8. Michael YU. Rachkov, Lino Marques, Anibal T. De Almeida, Multi-Sensing Demining Robot.

9. Zhenjun He, Jiang Zhang, Peng Xu, Jiaheng Qin and Yunkai Zhu, "Mine Detecting Robot Based on Wireless Communication with Multi-sensor",

10. Majd Ghareeb, Ali Bazzi, Mohamad Raad, Samih Abdul Nabi Wireless Robo-Pi for Landmine Detection 